\title{
Spin qubits in antidot lattices
}

Pedersen, Jesper Goor; Flindt, Christian; Mortensen, Niels Asger; Jauho, Antti-Pekka

\section{Published in:}

Physical Review B Condensed Matter

Link to article, DOI:

10.1103/PhysRevB.77.045325

Publication date:

2008

Document Version

Publisher's PDF, also known as Version of record

Link back to DTU Orbit

Citation (APA):

Pedersen, J. G., Flindt, C., Mortensen, N. A., \& Jauho, A-P. (2008). Spin qubits in antidot lattices. Physical Review B Condensed Matter, 77(4), 045325. https://doi.org/10.1103/PhysRevB.77.045325

\section{General rights}

Copyright and moral rights for the publications made accessible in the public portal are retained by the authors and/or other copyright owners and it is a condition of accessing publications that users recognise and abide by the legal requirements associated with these rights.

- Users may download and print one copy of any publication from the public portal for the purpose of private study or research.

- You may not further distribute the material or use it for any profit-making activity or commercial gain

- You may freely distribute the URL identifying the publication in the public portal

If you believe that this document breaches copyright please contact us providing details, and we will remove access to the work immediately and investigate your claim 


\title{
Spin qubits in antidot lattices
}

\author{
Jesper Pedersen, ${ }^{1}$ Christian Flindt, ${ }^{1}$ Niels Asger Mortensen, ${ }^{1}$ and Antti-Pekka Jauho ${ }^{1,2}$ \\ ${ }^{1}$ MIC - Department of Micro and Nanotechnology, NanoDTU, Technical University of Denmark, Building 345 East, \\ DK-2800 Kongens Lyngby, Denmark \\ ${ }^{2}$ Laboratory of Physics, Helsinki University of Technology, P. O. Box 1100, FI-02015 HUT, Finland \\ (Received 10 August 2007; revised manuscript received 28 September 2007; published 22 January 2008)
}

\begin{abstract}
We suggest and study designed defects in an otherwise periodic potential modulation of a two-dimensional electron gas as an alternative approach to electron spin based quantum information processing in the solid-state using conventional gate-defined quantum dots. We calculate the band structure and density of states for a periodic potential modulation, referred to as an antidot lattice, and find that localized states appear, when designed defects are introduced in the lattice. Such defect states may form the building blocks for quantum computing in a large antidot lattice, allowing for coherent electron transport between distant defect states in the lattice, and for a tunnel coupling of neighboring defect states with corresponding electrostatically controllable exchange coupling between different electron spins.
\end{abstract}

DOI: 10.1103/PhysRevB.77.045325

PACS number(s): 73.22.-f, 75.30.Et, 73.21.Cd, 03.67.Lx

\section{INTRODUCTION}

Localized electrons spins in a solid state structure have been suggested as a possible implementation of a future device for large-scale quantum information processing. ${ }^{1}$ Together with single spin rotations, the exchange coupling between spins in tunnel coupled electronic levels would provide a universal set of quantum gate operations. ${ }^{2} \mathrm{Re}-$ cently, both of these operations have been realized in experiments on electron spins in double quantum dots, demonstrating electron spin resonance (ESR) driven single spin rotations ${ }^{3}$ and electrostatic control of the exchange coupling between two electron spins. ${ }^{4}$ Combined with the long coherence time of the electron spin due to its weak coupling to the environment, and the experimental ability to initialize a spin and reading it out, ${ }^{5}$ four of DiVincenzo's five criteria ${ }^{6}$ for implementing a quantum computer may essentially be considered fulfilled. This leaves only the question of scalability experimentally unaddressed.

While large-scale quantum information processing with conventional gate-defined quantum dots is a topic of ongoing theoretical research, ${ }^{7}$ we here suggest and study an alternative approach based on so-called defect states that form at designed defects in a periodic potential modulation of a twodimensional electron gas (2DEG) residing at the interface of a semiconductor heterostructure. ${ }^{8}$ One way of implementing the potential modulation would be similar to the periodic antidot lattices ${ }^{9,10}$ that are now routinely fabricated. Such lattices can be fabricated on top of a semiconductor heterostructure using local oxidation techniques that allow for a precise patterning of arrays of insulating islands, with a spacing on the order of $100 \mathrm{~nm}$, in the underlying 2DEG. ${ }^{11}$ Even though the origin of these depletion spots is not essential for our proposal, we refer to them as antidots, and a missing antidot in the lattice as a defect. Alternative fabrication methods include electron beam and photolithography. ${ }^{12,13}$ In Ref. 11 a square lattice consisting of $20 \times 20=400$ antidots was patterned on an approximately $2.5 \mu \mathrm{m} \times 2.5 \mu \mathrm{m}$ area, and the available fabrication methods suggest that even larger antidot lattices with more than 1000 antidots and many defect states may be within experimental reach.
The idea of using designed defects in antidot lattices as a possible quantum computing architecture was originally proposed by some of us in Ref. 8, where we presented simple calculations of the single-particle level structure of an antidot lattice with one or two designed defects. Here, we take these ideas further and present detailed band structure and density of states calculations for a periodic lattice, describe a resonant tunneling phenomenon allowing for electron transport between distant defects in the lattice, and calculate numerically the exchange coupling between spins in two neighboring defects, showing that the suggested architecture could be useful for spin-based quantum information processing. The envisioned structure and the basic building blocks are shown schematically in Fig. 1.

The paper is organized as follows. In Sec. II we introduce our model of the antidot lattice and present numerical results for the band structure and density of states of a periodic
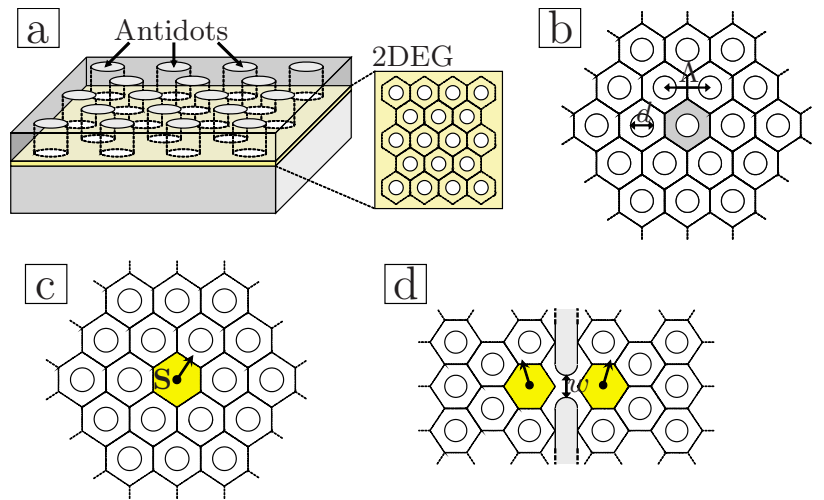

FIG. 1. (Color online) (a) Schematic illustration of a periodic antidot lattice; antidots may, e.g., be fabricated using local oxidation of a $\mathrm{Ga}[\mathrm{Al}] \mathrm{As}$ heterostructure. (b) Geometry of the periodic antidot lattice with the Wigner-Seitz cell marked in gray and the antidot diameter $d$ and lattice constant $\Lambda$ indicated. (c) A designed defect leads to the formation of defect states in which an electron with spin $\mathbf{S}$ can reside. (d) Tunnel coupled defects. The coupling can be controlled using a split-gate with an effective opening denoted $w$. 

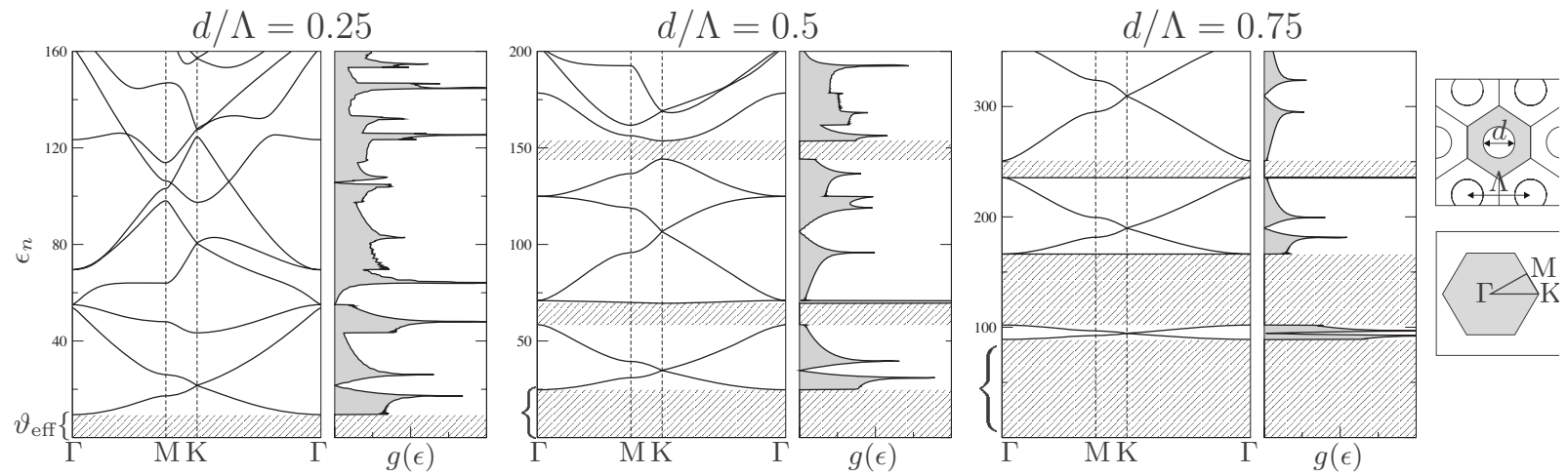

FIG. 2. Band structures and densities of states $g(\epsilon)$ of the periodic antidot lattice for three different values of the relative antidot diameter $d / \Lambda$. Notice the different energy scales for the three cases. On each band structure the gap $\vartheta_{\text {eff }}$ is indicated, below which no states exist for the periodic lattice. The band gaps and the gap below $\vartheta_{\text {eff }}$ are highlighted as hatched regions. Also shown is the periodic lattice structure with the Wigner-Seitz cell indicated in gray, as well as the first Brillouin zone (FBZ) with the three high-symmetry points and the irreducible FBZ indicated.

antidot lattice. In particular, we show that the periodic potential modulation gives rise to band gaps in the otherwise parabolic free electron band structure. In Sec. III we introduce a single missing antidot, a defect, in the lattice and calculate numerically the eigenvalue spectrum of the localized defect states that form at the location of the defect. We develop a semianalytic model that explains the level structure of the lowest-lying defect states. In Sec. IV we consider two neighboring defect states and calculate numerically the tunnel coupling between them. In Sec. V we describe a principle for coherent electron transport between distant defect states in the antidot lattice, and illustrate this phenomenon by wave packet propagations. In Sec. VI we present numerically exact results for the exchange coupling between electron spins in tunnel coupled defect states, before we finally in Sec. VII present our conclusions.

\section{PERIODIC ANTIDOT LATTICE}

We first consider a triangular lattice of antidots with lattice constant $\Lambda$ superimposed on a two-dimensional electron gas (2DEG). The structure is shown schematically together with the Wigner-Seitz cell in Fig. 1(b). While experiments on antidot lattices are often performed in a semiclassical regime, where the typical feature sizes and distances, e.g., the lattice constant $\Lambda$, are much larger than the electron wavelength, we here consider the opposite regime, where these length scales are comparable, and a full quantum-mechanical treatment is necessary. In the effective-mass approximation we thus model the periodic lattice with a two-dimensional singleelectron Hamiltonian reading

$$
H=-\frac{\hbar^{2}}{2 m^{*}} \nabla_{\mathbf{r}}^{2}+\sum_{i} V\left(\mathbf{r}-\mathbf{R}_{i}\right), \quad \mathbf{r}=(x, y),
$$

where $m^{*}$ is the effective mass of the electron and $V\left(\mathbf{r}-\mathbf{R}_{i}\right)$ is the potential of the $i$ th antidot positioned at $\mathbf{R}_{i}$. We model each antidot as a circular potential barrier of diameter $d$ so that $V\left(\mathbf{r}-\mathbf{R}_{i}\right)=V_{0}$ for $\left|\mathbf{r}-\mathbf{R}_{i}\right| \leq d / 2$ and zero otherwise. In the limit $V_{0} \rightarrow \infty$ the eigenfunctions do not penetrate into the antidots, and the Schrödinger equation may be written as

$$
-\Lambda^{2} \nabla_{\mathbf{r}}^{2} \psi_{n}(\mathbf{r})=\epsilon_{n} \psi_{n}(\mathbf{r})
$$

with the boundary condition $\psi_{n}=0$ in the antidots, and where we have introduced the dimensionless eigenvalues

$$
\epsilon_{n}=E_{n} \Lambda^{2} 2 m^{*} / \hbar^{2} \text {. }
$$

In the following we use parameter values typical of GaAs, for which $\hbar^{2} / 2 m^{*} \simeq 0.6 \mathrm{eV} \mathrm{nm}{ }^{2}$ with $m^{*}=0.067 m_{e}$, although the choice of material is not essential. We have checked numerically that our results are not critically sensitive to the approximation $V_{0} \rightarrow \infty$, so long as the height is significantly larger than any energies under consideration. All results presented in this work have thus been calculated in this limit, for which the simple form of the Schrödinger equation Eq. (2) applies. In this limit, the band structures presented below are of a purely geometrical origin. The band structure can be calculated by imposing periodic boundary conditions and solving Eq. (2) on the finite domain of the Wigner-Seitz cell. We solve this problem using a finite-element method. ${ }^{14}$ The corresponding density of states is calculated using the linear tetrahedron method in its symmetry corrected form. ${ }^{15-17}$

In Fig. 2 we show the band structure and density of states of the periodic antidot lattice for three different values of the relative antidot diameter $d / \Lambda$. We note that an increasing antidot diameter raises the kinetic energy of the Bloch states due to the increased confinement and that several band gaps open up. We have indicated the gap $\vartheta_{\text {eff }}$ below which no states exist for the periodic structure. We shall denote as band gaps only those gaps occurring between two bands, and thus we do not refer to the gap below $\vartheta_{\text {eff }}$ as a band gap in the following. This is motivated by the difference in the underlying mechanisms responsible for the gaps: While the band gaps rely on the periodicity of the antidot lattice, similar to Bragg reflection in the solid state, the gap below $\vartheta_{\text {eff }}$ represents an averaging of the potential landscape generated by the antidots, and is thus robust against lattice disorder as we have also checked numerically. ${ }^{18}$ The lowest band gap is thus present for $d / \Lambda>0.35$ while the higher-energy band gap only develops for $d / \Lambda>0.45$. As the antidot diameter is 
increased, several flat bands appear with $\nabla_{\mathbf{k}} \epsilon_{n}(\mathbf{k}) \simeq 0$, giving rise to van Hove singularities in the corresponding density of states.

\section{DEFECT STATES}

We now introduce a defect in the lattice by leaving out a single antidot. Topologically, this structure resembles a planar 2D photonic crystal, and relying on this analogy we expect one or more localized defect states to form inside the defect. ${ }^{19}$ The gap $\vartheta_{\text {eff }}$ indicated in Fig. 2 may be considered as the height of an effective two-dimensional potential surrounding the defect, and thus gives an upper limit to the existence of defect states in this gap. Similar states are expected to form in the band gaps of the periodic structure, which are highlighted in Fig. 2. As defect states decay to zero far from the location of the defect, we have a large freedom in the way we spatially truncate the problem at large distances. For simplicity we use a super-cell approximation, but with $\psi=0$ imposed on the edge, thus leaving Eq. (2) a Hermitian eigenvalue problem which we may conveniently solve with a finite-element method. ${ }^{14}$ Other choices, such as periodic boundary conditions, do not influence our numerical results. The size of the supercell has been chosen sufficiently large, such that the results are unaffected by a further increase in size.

In the insets of Fig. 3(a) we show the calculated eigenfunctions corresponding to the two lowest energy eigenvalues for a relative antidot diameter $d / \Lambda=0.5$. As expected, we find that defect states form that to a high degree are localized within the defect. The second-lowest eigenvalue is twofold degenerate and we only show one of the corresponding eigenstates. The figure shows the energy eigenvalues of the defect states as a function of the relative antidot diameter together with the gap $\vartheta_{\text {eff }}$. As this effective potential is increased, additional defect states become available and we may thus tune the number of levels in the defect by adjusting the relative antidot diameter. In particular, we note that for $d / \Lambda \lesssim 0.42$ only a single defect state forms. As the sizes of the antidots are increased, the confinement of the defect states becomes stronger, leading to an increase in their energy eigenvalues. For GaAs with $d / \Lambda=0.5$ and $\Lambda=75 \mathrm{~nm}$ the energy splitting of the two lowest defect states is approximately $1.1 \mathrm{meV}$, which is much larger than $k_{B} T$ at subKelvin temperatures, and the level structure is thus robust against thermal dephasing.

In Fig. 3(b) we show similar results for defect states residing in the lowest band gap of the periodic structure. While the states residing below $\vartheta_{\text {eff }}$ resemble those occurring due to the confining potential in conventional gate-defined quantum dots, these higher-lying states are of a very different nature, being dependent on the periodicity of the surrounding lattice. For the band gaps, the existence of bound states is limited by the relevant band edges as indicated in the figure. As the size of the band gap is increased, additional defect states become available and we may thus also tune the number of levels residing in the band gaps by adjusting the relative antidot diameter.

Because the formation of localized states residing below $\vartheta_{\text {eff }}$ depends only on the existence of the effective potential
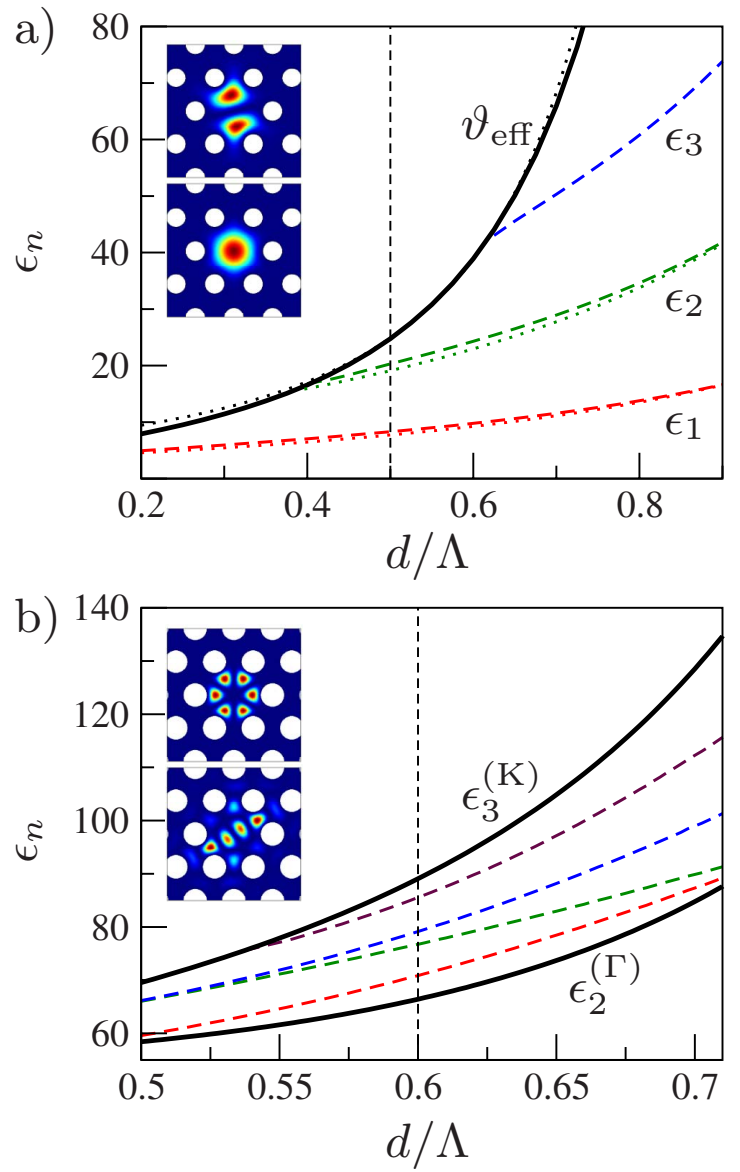

FIG. 3. (Color online) Energy spectrum for a single defect. The (dimensionless) eigenvalues corresponding to localized states are shown as a function of the relative antidot diameter $d / \Lambda$. For a given choice of $\Lambda$, the eigenvalues can be converted to meV using Eq. (3). (a) Energy spectrum for defect states residing in the gap below $\vartheta_{\text {eff. }}$ The full line indicates the height $\vartheta_{\text {eff }}$ of the effective potential in which the localized states reside. The dotted lines are the approximate expressions given by Eqs. (4), (6), and (7). The approximate results for $\epsilon_{1}$ are in almost perfect agreement with the numerical calculations. (b) Energy spectrum for the defect states residing in the lowest band gap region. The full lines indicate the band gap edges of the periodic structure, $\epsilon_{3}^{(K)}$ and $\epsilon_{2}^{(\Gamma)}$, giving upper and lower limits to the existence of bound states. The inset in both figures show the localized states corresponding to the two lowest energy eigenvalues indicated by the dashed vertical lines. The absolute square is shown.

surrounding the defect, the formation of such states is not critically dependent on perfect periodicity of the surrounding lattice, which we have checked numerically. ${ }^{18}$ Also, the lifetimes of the states due to the finite size of the antidot lattice are of the order of seconds even for a relatively small number of rings of antidots surrounding the defect. ${ }^{8}$ However, the localized states residing in the band gaps are more sensitive to lattice disorder, since they rely more crucially on the periodicity of the surrounding lattice. Introducing disorder may induce a finite density of states in the band gaps of the periodic structure and thus significantly decrease the lifetimes of the localized states residing in this region. 
In order to gain a better understanding of the levelstructure of the defect states confined by $\vartheta_{\text {eff }}$ we develop a semianalytic model for $\vartheta_{\text {eff }}$ and the corresponding defect states. We first note that the effective potential $\vartheta_{\text {eff }}$ is given by the energy of the lowest Bloch state at the $\Gamma$ point of the periodic lattice. At this point $\mathbf{k}=\mathbf{0}$ and Bloch's theorem reduces to an ordinary Neumann boundary condition on the edge of the Wigner-Seitz cell. This problem may be solved using a conformal mapping, and we obtain the expression ${ }^{20}$

$$
\vartheta_{\text {eff }} \simeq\left(\mathcal{C}_{1}+\frac{\mathcal{C}_{2}}{\mathcal{C}_{3}-d / \Lambda}\right)^{2}
$$

where $\mathcal{C}_{1} \simeq-0.2326, \mathcal{C}_{2} \simeq 2.7040$, and $\mathcal{C}_{3} \simeq 1.0181$ are given by expressions involving the Bessel functions $Y_{0}$ and $Y_{1}$. We now consider the limit of $d / \Lambda \rightarrow 1$ and note that in this case the defect states residing below $\vartheta_{\text {eff }}$ are subject to a potential which we may approximate as an infinite two-dimensional spherical potential well with radius $\Lambda-d / 2$. The lowest eigenvalue for this problem is $\epsilon_{1}^{(\infty)}=\Lambda^{2} \alpha_{0,1}^{2} /(\Lambda-d / 2)^{2}$, where $\alpha_{0,1} \simeq 2.405$ is the first zero of the zeroth order Bessel function. This expression yields the correct scaling with $d / \Lambda$, but is only accurate in the limit of $d / \Lambda \rightarrow 1$. We correct for this by considering the limit of $d / \Lambda \rightarrow 0$, in which we may solve the problem using ideas developed by Glazman et al. in studies of quantum conductance through narrow constrictions. ${ }^{21}$ The problem may be approximated as a two-dimensional spherical potential well of height $\pi^{2}$ and radius $\Lambda$. The lowest eigenvalues $\epsilon_{1}^{\left(\pi^{2}\right)}$ of this problem is the first root of the equation

$$
\sqrt{\epsilon_{1}^{\left(\pi^{2}\right)}} \frac{J_{1}\left(\sqrt{\epsilon_{1}^{\left(\pi^{2}\right)}}\right)}{J_{0}\left(\sqrt{\left.\epsilon_{1}^{\left(\pi^{2}\right)}\right)}\right.}=\sqrt{\pi^{2}-\epsilon_{1}^{\left(\pi^{2}\right)}} \frac{K_{1}\left(\sqrt{\pi^{2}-\epsilon_{1}^{\left(\pi^{2}\right)}}\right)}{K_{0}\left(\sqrt{\pi^{2}-\epsilon_{1}^{\left(\pi^{2}\right)}}\right)},
$$

where $J_{i}\left(K_{i}\right)$ is the $i$ th order Bessel function of the first (second) kind. If the height of the potential well $\pi^{2}$ is much larger than the energy eigenvalues, the first root would simply be $\alpha_{0,1}^{2}$. Lowering the confinement must obviously shift down the eigenvalue, and in the present case we find that $\epsilon_{1}^{\left(\pi^{2}\right)} \simeq \pi$. By expanding the equation to first order in $\sqrt{\epsilon_{1}^{\left(\pi^{2}\right)}}$ around $\sqrt{\pi}$ we may solve the equation to obtain $\epsilon_{1}^{\left(\pi^{2}\right)}$ $\simeq 3.221$, which is in excellent agreement with a full numerical solution of Eq. (5). Correcting for the low- $d / \Lambda$ behavior we thus find the approximate expression for the lowest energy eigenvalue ${ }^{8}$

$$
\epsilon_{1} \simeq \epsilon_{1}^{(\infty)}-\lim _{d / \Lambda \rightarrow 0} \epsilon_{1}^{(\infty)}+\epsilon_{1}^{\left(\pi^{2}\right)}=\epsilon_{1}^{\left(\pi^{2}\right)}+\frac{(4-d / \Lambda) d / \Lambda}{(2-d / \Lambda)^{2}} \alpha_{0,1}^{2}
$$

A similar analysis leads to an approximate expression for the first excited state $\epsilon_{2}$. This mode has a finite angular momentum of \pm 1 and a radial $J_{1}$ solution yields

$$
\epsilon_{2} \simeq \epsilon_{2}^{\left(\pi^{2}\right)}+\frac{(4-d / \Lambda) d / \Lambda}{(2-d / \Lambda)^{2}} \alpha_{1,1}^{2}
$$

where $\epsilon_{2}^{\left(\pi^{2}\right)} \simeq 7.673$ is the second-lowest eigenvalue of the two-dimensional spherical potential well of height $\pi^{2}$ and radius $\Lambda$, which can be found from an equation very similar to Eq. (5). The first root of the first-order Bessel function is $\alpha_{1,1} \simeq 3.832$. The scaling of the two lowest eigenvalues with $d / \Lambda$ is thus approximately the same. The approximate expressions are indicated by the dotted lines in Fig. 3, and we note an excellent agreement with the numerical results. We remark that the filling of the defect states can be controlled using a metallic back gate that changes the electron density and thus the occupation of the different defect states. ${ }^{22}$

\section{TUNNEL COUPLED DEFECT STATES}

Two closely situated defect states can have a finite tunnel coupling, leading to the formation of hybridized defect states. The coupling between the two defects may be tuned via a metallic split gate defined on top of the $2 \mathrm{DEG}$ in order to control the opening between the two defects. As the voltage is increased the opening is squeezed, leading to a reduced overlap between the defect states. We model such a split gate as an infinite potential barrier shaped as shown in Fig. 1(d). Changing the applied voltage effectively leads to a change in the relative width $w / \Lambda$ of the opening, which we take as a control parameter in the following. If we consider just a single level in each defect we can calculate the tunnel matrix element as $|\tau|=\left(\epsilon_{+}-\epsilon_{-}\right) / 2$ where $\epsilon_{ \pm}$are the eigenenergies of the bonding and antibonding states, respectively, of the double defect. In the following, we calculate the tunnel coupling between two defect states lying below $\vartheta_{\text {eff }}$, but the analysis applies equally well to defect states lying in the band gaps.

In Fig. 4 we show the tunnel matrix element $|\tau|$ as a function of the relative gate constriction width $w / \Lambda$ for three different values of $d / \Lambda$ in the single-level regime of each defect, i.e., $d / \Lambda \lesssim 0.42$. As expected, the tunnel coupling grows with increasing constriction width due to the increased overlap between the defect states. A saturation point is reached when the constriction width is on the order of the diameter of the defect states, after which the overlap is no longer increased significantly. An electron prepared in one of the defect states will oscillate coherently between the two defect states with a period given as $T=\pi \hbar /|\tau|$, which for GaAs with $\Lambda=75 \mathrm{~nm}, d / \Lambda=0.4$, and $w / \Lambda=0.6$ implies an oscillation time of $T \simeq 0.14$ ns. A numerical wave packet propagation of an electron initially prepared in the left defect state is shown in Fig. 4(b), confirming the expected oscillatory behavior. With a finite tunnel coupling between two defect states, two electron spins trapped in the defects will interact due to the exchange coupling, to which we return in Sec. VI.

\section{RESONANT COUPLING OF DISTANT DEFECT STATES}

With a large antidot lattice and several defect states it may be convenient with quantum channels along which coherent electron transport can take place, connecting distant defect states. In Refs. 23 and 24 it was suggested to use arrays of tunnel coupled quantum dots as a means to obtain highfidelity electron transfer between two distant quantum dots. We have applied this idea to an array of tunnel coupled de- 


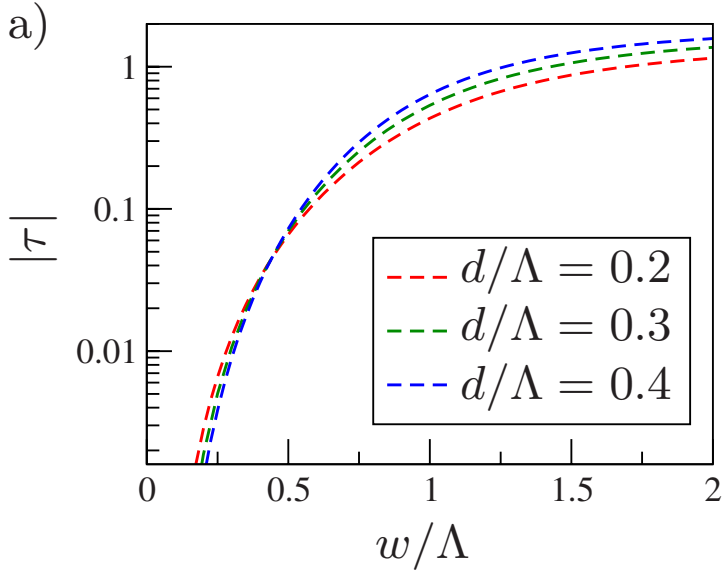

b)

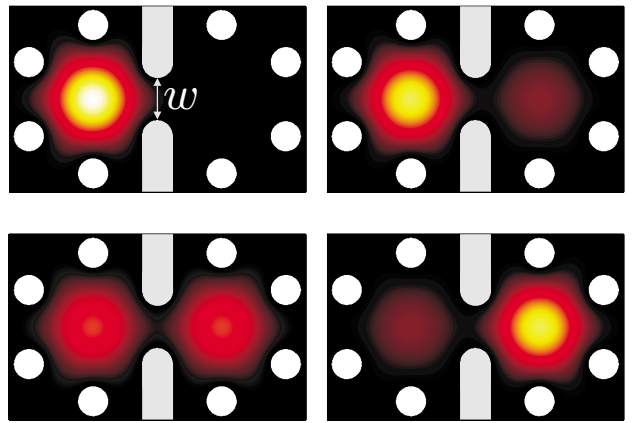

FIG. 4. (Color online) (a) The (dimensionless) tunnel coupling $|\tau|$ as a function of the relative split gate constriction width $w / \Lambda$ for three different values of $d / \Lambda$ in the single-level regime. For a given choice of $\Lambda$, the tunnel couplings can be converted to $\mathrm{meV}$ using Eq. (3). (b) Time propagation of an electron initially prepared in the left defect state for $d / \Lambda=0.4$ and $w / \Lambda=0.6$. The absolute square of the initial wave function is shown in the upper left panel. The following panels show the state after a time span of $T / 8,2 T / 8$, and $3 T / 8$, respectively, where $T$ is the oscillation period.

fect states and confirmed that this mechanism may be used for coherent electron transport between distant defects in an antidot lattice. ${ }^{18}$ This approach, however, relies on precise tunings of the tunnel couplings between each defect in the array, which may be difficult to implement experimentally. Instead, we suggest an alternative approach based on a resonant coupling phenomenon inspired by similar ideas used to couple light between different fiber cores in a photonic crystal fiber. ${ }^{25,26}$

We consider two defects separated by a central line of $N$ antidots and a central back gate $V_{g}$ in the region between the defects, as shown in Fig. 5. Again, we consider defect states residing below $\vartheta_{\text {eff }}$, but the principle described here may equally well be applied to defect states in the band gaps. Using the back gate, the potential between the two defects can be controlled locally. If the potential is lowered below $\vartheta_{\text {eff }}$, a discrete spectrum of standing-wave solutions forms between the two defects. In the following we denote the energy of one of these standing-wave solutions by $\epsilon_{g}$, while the energy of the two defect states is assumed to be identical and is denoted $\epsilon_{d}$. A simple three-level analysis of this system, as illustrated in Fig. 5, reveals that by tuning the back
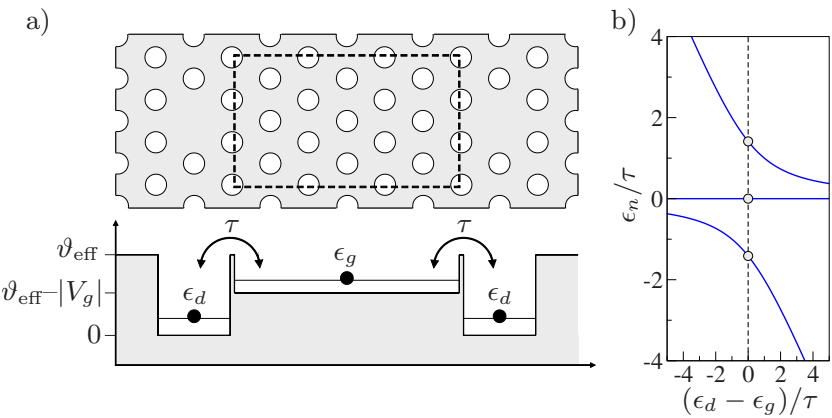

FIG. 5. (Color online) (a) The structure considered for resonant coupling of distant defect states; two defects separated by a central line of $N=3$ antidots, with a central back gate $V_{g}$ controlling the potential square well in the region marked with dashed lines. A simple three-level model of the system is illustrated below. (b) The eigenvalue spectrum of the three-level model. The dashed line marks the point of resonance.

gate so that the levels are aligned, $\epsilon_{g}=\epsilon_{d}$, a resonant coupling between the two distant defects occurs, characterized by a symmetric splitting of the three lowest eigenvalues into $\epsilon_{0}$ $=\epsilon_{d}$ and $\epsilon_{ \pm}=\epsilon_{d} \pm \sqrt{2}|\tau|$, where $|\tau|$ is the tunnel coupling between the defects and the standing-wave solution in the central back gate region. If an electron is prepared in one of the defects states, it will oscillate coherently between the two defects with an oscillation period of $T=\sqrt{2} \pi \hbar / \mid \tau$. By turning off the back gate at time $t=T / 2$ we may thereby trap the electron in the opposite defect which may by situated a distance an order of magnitude larger than the lattice constant away from the other defect.

In Fig. 6 we show the numerically calculated eigenvalues as a function of the depth $\left|V_{g}\right|$ of the central potential square well of the structure illustrated in Fig. 5 for $d / \Lambda=0.5$ and a central line of $N=7$ antidots separating the two defects. Contrary to the simple three-level model, several resonances now occur as the back gate is lowered, corresponding to coupling

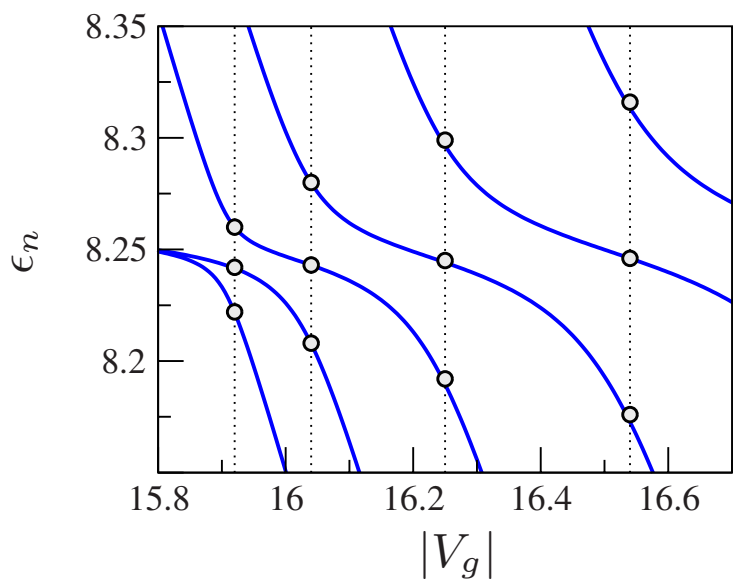

FIG. 6. (Color online) Energy eigenvalues as a function of the magnitude $\left|V_{g}\right|$ of the back gate for the structure illustrated in Fig. 5 for $d / \Lambda=0.5$ and a central line of $N=7$ antidots separating the two defects. The resonances are marked with dotted lines and characterized by a symmetric splitting of the eigenvalues. 

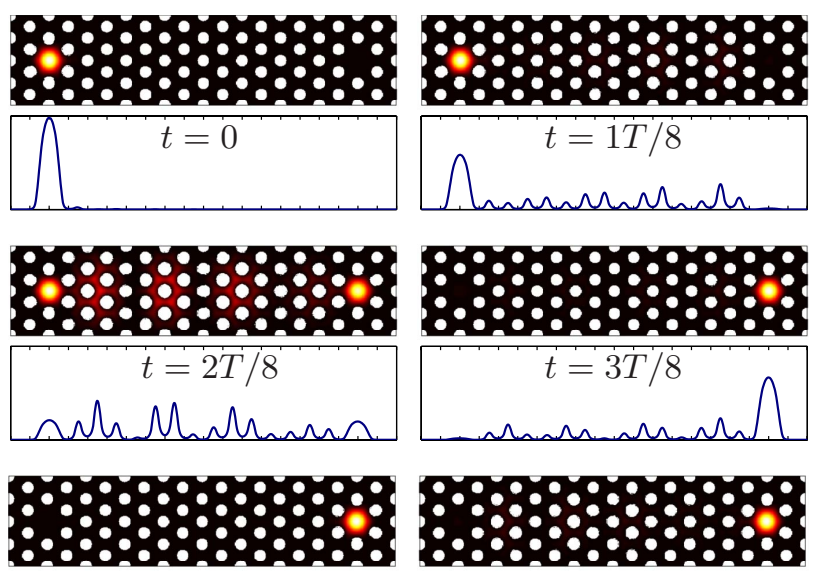

$t=4 T / 8$
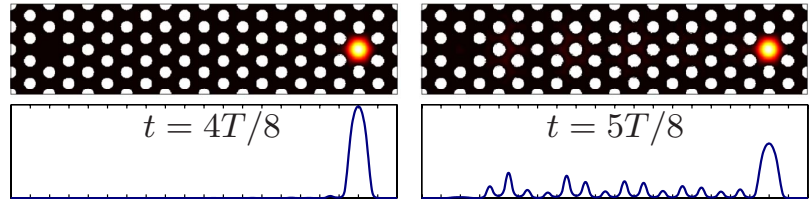

FIG. 7. (Color online) Numerical time propagation of an electron initially prepared in the left defect of the structure illustrated in Fig. 5(a) and corresponding to the results of Fig. 6 with $\left|V_{\mathrm{g}}\right|$ $\simeq 16.54$. The charge densities $\rho(x, y)$ are shown in the upper panels, while the lower panels show $\int d y \rho(x, y)$. The oscillation period is denoted $T$.

to different standing-wave solutions in the multileveled central region. The energy splitting at resonance is larger when the defect states couple to higher-lying central states due to a large overlap between the defect states and the central standing-wave solution. In Fig. 7 we show a numerical time propagation of an electron initially prepared in the left defect, confirming the oscillatory behavior expected from the simple model. For GaAs and $\Lambda=75 \mathrm{~nm}$ the results indicate an oscillation period of $T \simeq 0.16 \mathrm{~ns}$ for the time propagation illustrated. The resonant phenomenon relies solely on the level alignment $\epsilon_{g}=\epsilon_{d}$ and on the symmetry condition that both defect states have the same energy and magnitude of tunnel coupling to the standing wave solution in the central region. It is in principle independent of the number of antidots $N$ separating the two defects, but in practice this range is limited by the coherence length of the sample and the fact that the levels of the central region grow too dense if $N$ becomes large. ${ }^{27}$ We have checked numerically that resonant coupling of defect levels below $\vartheta_{\text {eff }}$ is robust against lattice disorder. ${ }^{18}$

\section{EXCHANGE COUPLING}

So far we have only considered the single-particle electronic level-structure of the antidot lattice. However, as mentioned in the Introduction, the exchange coupling between electron spins is a crucial building block for a spin based quantum computing architecture, and in fact suffices to implement a universal set of quantum gates. ${ }^{28}$ The exchange coupling is a result of the Pauli principle for identical fermions, which couples the symmetries of the orbital and spin degrees of freedom. If the orbital wave function of the two electrons is symmetric (i.e., preserves sign under particle exchange), the spins must be in the antisymmetric singlet state, while an antisymmetric orbital wave function means that the spins are in a symmetric triplet state. One may thereby map the splitting between the ground state energy $E_{S}$ of the symmetric orbital subspace and the ground state energy $E_{A}$ of the antisymmetric orbital subspace onto an effective Heisenberg spin Hamiltonian $\mathcal{H}=J \mathbf{S}_{1} \cdot \mathbf{S}_{2}$, where $J=E_{A}-E_{S}$ is the exchange coupling between the two spins $\mathbf{S}_{1}$ and $\mathbf{S}_{2}$. The implementation of quantum gates based on the exchange coupling requires that $J$ can be varied over several orders of magnitude in order to effectively turn the coupling on and off. In this section we present numerically exact results for the exchange coupling between two electron spins residing in tunnel coupled defects as those illustrated in Fig. 1(d).

The Hamiltonian of two electrons in two tunnel coupled defects may be written as

$$
H\left(\mathbf{r}_{1}, \mathbf{r}_{2}\right)=h\left(\mathbf{r}_{1}\right)+h\left(\mathbf{r}_{2}\right)+C\left(\mathbf{r}_{1}, \mathbf{r}_{2}\right),
$$

where

$$
C\left(\mathbf{r}_{1}, \mathbf{r}_{2}\right)=\frac{e^{2}}{4 \pi \epsilon_{r} \epsilon_{0}} \frac{1}{\left|\mathbf{r}_{1}-\mathbf{r}_{2}\right|}
$$

is the Coulomb interaction and the single-electron Hamiltonians are

$$
h\left(\mathbf{r}_{i}\right)=\frac{\left(\mathbf{p}_{i}+e \mathbf{A}\right)^{2}}{2 m^{*}}+V\left(\mathbf{r}_{i}\right)+\frac{1}{2} g \mu_{B} B S_{z, i}, \quad i=1,2,
$$

where $V(\mathbf{r})$ is the potential due to the antidots and the coupled defects. As previously, we model the antidots and the split gate as potential barriers of infinite height, and use finite-element methods to solve the single-electron problem defined by Eq. (10). A Zeeman field $B \hat{\mathbf{z}}$ applied perpendicularly to the electron gas splits the spin states, and we choose a corresponding vector potential reading $\mathbf{A}=B(-y \hat{\mathbf{x}}+x \hat{\mathbf{y}}) / 2$.

In order to calculate the exchange coupling $J$ we employ a recently developed method for numerically exact finiteelement calculations of the exchange coupling: ${ }^{29}$ The full two-electron problem is solved by expressing the twoelectron Hamiltonian in a basis of product states of singleelectron solutions obtained using a finite element method. ${ }^{14}$ The Coulomb matrix elements are evaluated by expanding the single-electron states in a basis of 2D Gaussians, ${ }^{30}$ and the two-particle Hamiltonian matrix resulting from this procedure may then be diagonalized in the subspaces spanned by the symmetric and antisymmetric product states, respectively, to yield the exchange coupling. The details of the numerical method are described elsewhere. ${ }^{18,29}$ The results presented below have all been obtained with a sufficient size of the 2D Gaussian basis set as well as the number of singleelectron eigenstates, such that a further increase does not change the results. ${ }^{31}$

In Fig. 8 we show the calculated exchange coupling for a double defect structure. The exchange coupling varies by several orders of magnitude as the split gate constriction width is increased, showing that electrostatic control of the exchange coupling in an antidot lattice is possible, similarly to the principles proposed ${ }^{2}$ and experimentally realized ${ }^{4}$ for double quantum dots. Just as the tunnel coupling, the exchange coupling reaches a saturation point when the split 

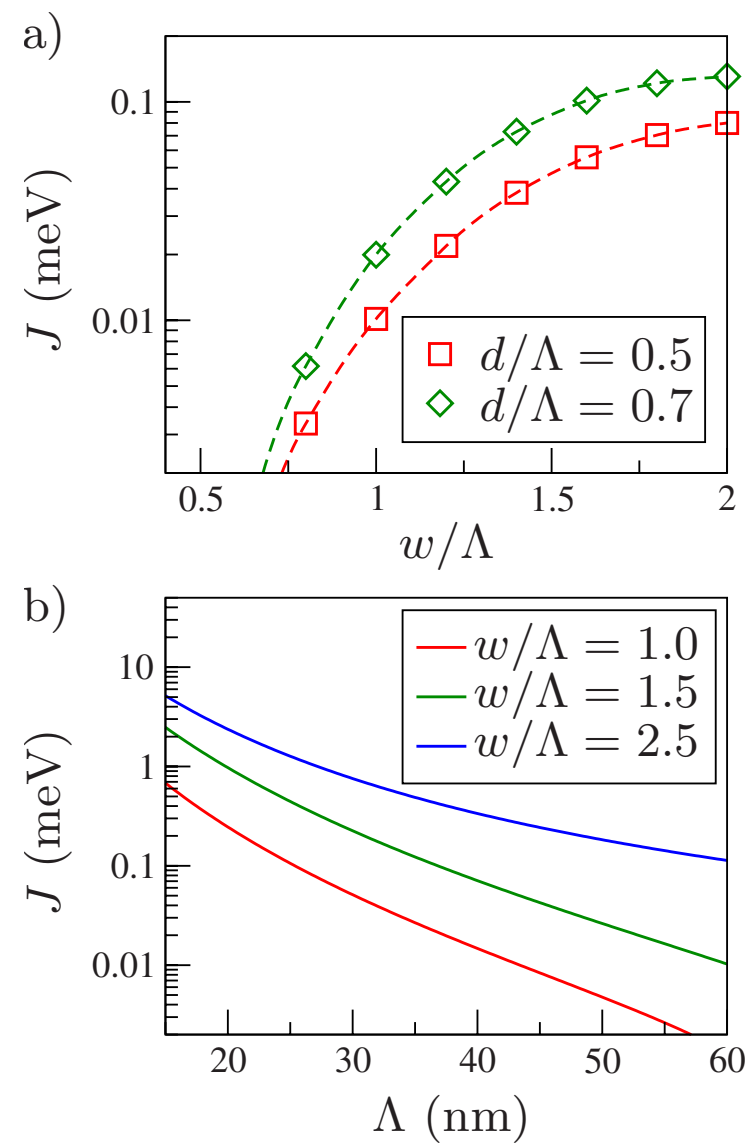

FIG. 8. (Color online) Exchange coupling $J$ for a double defect structure. (a) Exchange coupling as a function of the relative split gate constriction width $w / \Lambda$ for two different values of the relative antidot diameter and a lattice constant $\Lambda=45 \mathrm{~nm}$. (b) Exchange coupling as a function of the lattice constant $\Lambda$ for three different values of the relative split gate constriction width.

gate constriction width is on the order of the diameter of the defect states. This is to be expected since the exchange coupling in the Hubbard approximation is proportional to the square of the tunnel coupling. ${ }^{2}$ As illustrated in Fig. 8(b), the exchange coupling is highly dependent on the lattice constant, increasing several orders of magnitude as the lattice constant is decreased from 60 to $20 \mathrm{~nm}$. This is in part due to the overall increase in the energies of the eigenstastes and the splitting between them with increased confinement, but also due to a decrease in the ratio of the Coulomb interaction strength to the confinement strength. As the relative strength of the Coulomb interaction is decreased, the defect states are effectively moved closer together, resulting in an increase in the exchange coupling.

The exchange coupling is also highly dependent on magnetic fields applied perpendicularly to the plane of the electrons. ${ }^{2}$ In Fig. 9 we show the exchange coupling as a function of $\omega_{c} / \omega_{0}$ where $\omega_{c}=e B / m^{*} c$ and we define $\omega_{0}$ $=\frac{\hbar}{2 m^{*} \Lambda^{2}}$. For GaAs $\omega_{c} / \omega_{0} \simeq 0.00104 \mathrm{~T}^{-1} \mathrm{~nm}^{-2} \Lambda^{2} B$. As expected, the results of Fig. 9 are very similar to those obtained for double quantum dots. ${ }^{2,30}$ In all cases we note an initial transition from the antiferromagnetic $(J>0)$ to the ferromagnetic $(J<0)$ regime of exchange coupling, followed by a
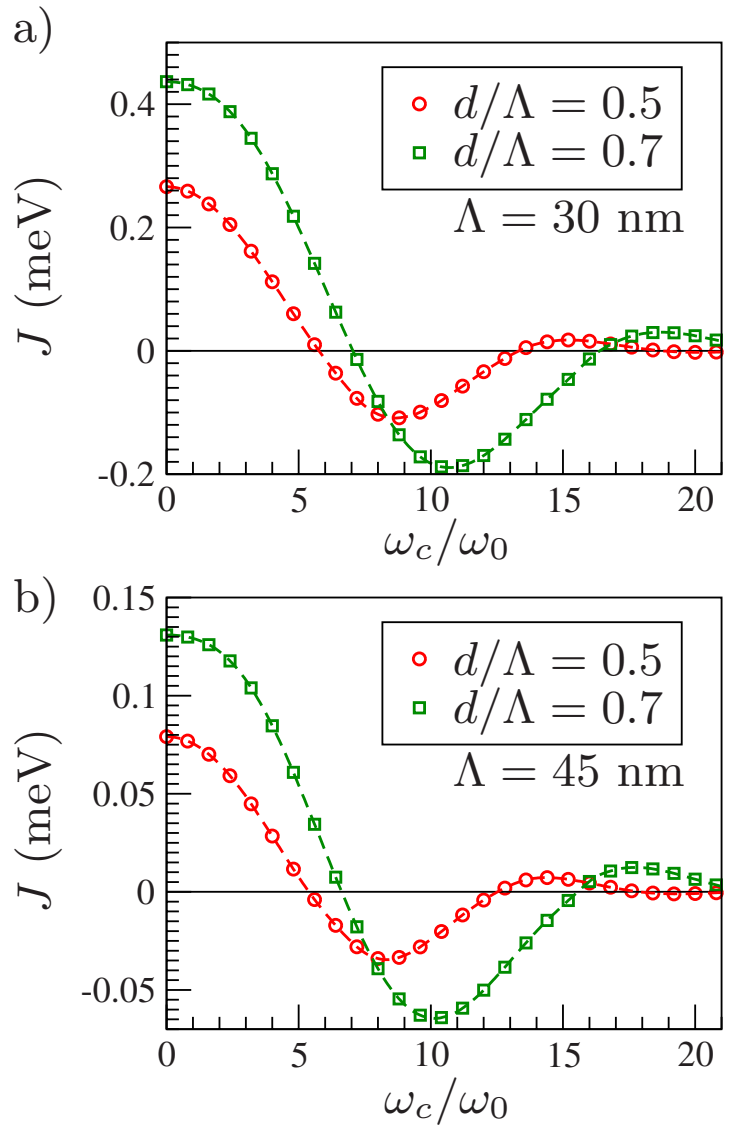

FIG. 9. (Color online) Exchange coupling $J$ for a double defect structure as a function of $\omega_{c} / \omega_{0}$, where $\omega_{c}=e B / m^{*} c$ and $\omega_{0}$ $=\hbar /\left(2 m^{*} \Lambda^{2}\right)$. Results are shown for a relative split gate constriction width $w / \Lambda=2$, and two different values of the relative antidot diameter $d / \Lambda$. The lattice constant is (a) $\Lambda=30 \mathrm{~nm}$ and (b) $\Lambda$ $=45 \mathrm{~nm}$.

return to positive values of the exchange coupling at higher magnetic fields. The initial transition to negative exchange coupling is caused by long-range Coulomb interactions. ${ }^{2}$ As the magnetic field is increased further, magnetic confinement becomes dominant, compressing the orbits and thus reducing the overlap between the single-defect wave functions. This leads to a strong reduction of the magnitude of the exchange coupling. Due to the increased confinement strength for smaller lattice constants $\Lambda$, these transitions occur at larger magnetic fields. The same is the case for the larger relative antidot diameters, in which the ratio of magnetic confinement to confinement due to the antidots is reduced. We have only considered the case of a large constriction width $w / \Lambda$ $=2$, since this regime of relatively large exchange coupling is the most interesting for practical purposes. For small values of $w / \Lambda$ we expect to find results similar to those obtained in the limit of large interdot distances for double quantum dot systems. $^{2}$

\section{CONCLUSIONS}

In conclusion, we have suggested and studied an alternative candidate for spin based quantum information process- 
ing in the solid-state, namely, defect states forming at the location of designed defects in an otherwise periodic potential modulation of a two-dimensional electron gas, here referred to as an antidot lattice. We have performed numerical band structure and density of states calculations of a periodic antidot lattice, and shown how localized defect states form at the location of designed defects. The antidot lattice allows for resonant coupling of distant defect states, enabling coherent transport of electrons between distant defects. Finally, we have shown that electrostatic control of the exchange coupling between electron spins in tunnel coupled defect states is possible, which is an essential ingredient for spin based quantum computing. Altogether, we believe that designed defects in antidot lattices provide several prerequisites for a large quantum information processing device in the solid state.

\section{ACKNOWLEDGMENTS}

We thank A. Harju for helpful advice during the development of our numerical routines, and T. G. Pedersen for fruitful discussions during the preparation of this manuscript. A.P.J. is grateful to the FiDiPro program of the Finnish Academy for support during the final stages of this work.
${ }^{1}$ D. Loss and D. P. DiVincenzo, Phys. Rev. A 57, 120 (1998).

${ }^{2}$ G. Burkard, D. Loss, and D. P. DiVincenzo, Phys. Rev. B 59, 2070 (1999).

${ }^{3}$ F. H. L. Koppens, C. Buizert, K. J. Tielrooij, I. T. Vink, K. C. Nowack, T. Meunier, L. P. Kouwenhoven, and L. M. K. Vandersypen, Nature (London) 442, 766 (2006).

${ }^{4}$ J. R. Petta, A. C. Johnson, J. M. Taylor, E. A. Laird, A. Yacoby, M. D. Lukin, C. M. Marcus, M. P. Hanson, and A. C. Gossard, Science 309, 2180 (2005).

${ }^{5}$ J. M. Elzerman, R. Hanson, L. H. W. van Beveren, B. Witkamp, L. M. K. Vandersypen, and L. P. Kouwenhoven, Nature (London) 430, 431 (2004).

${ }^{6}$ D. P. DiVincenzo, Fortschr. Phys. 48, 771 (2000).

${ }^{7}$ J. M. Taylor, H. A. Engel, W. Dur, A. Yacoby, C. M. Marcus, P. Zoller, and M. D. Lukin, Nat. Phys. 1, 177 (2005).

${ }^{8}$ C. Flindt, N. A. Mortensen, and A. P. Jauho, Nano Lett. 5, 2515 (2005)

${ }^{9}$ K. Ensslin and P. M. Petroff, Phys. Rev. B 41, 12307 (1990).

${ }^{10}$ D. Weiss, K. Richter, A. Menschig, R. Bergmann, H. Schweizer, K. von Klitzing, and G. Weimann, Phys. Rev. Lett. 70, 4118 (1993).

${ }^{11}$ A. Dorn, E. Bieri, T. Ihn, K. Ensslin, D. D. Driscoll, and A. C. Gossard, Phys. Rev. B 71, 035343 (2005).

${ }^{12}$ Y. Luo and V. Misra, Nanotechnology 17, 4909 (2006).

${ }^{13}$ J. I. Martín, J. Nogués, K. Liu, J. L. Vicent, and I. K. Schuller, J. Magn. Magn. Mater. 256, 449 (2003).

${ }^{14}$ We have used the COMSOL Multiphysics 3.2 package for all finiteelement calculations. See www.comsol.com. Convergence with respect to the mesh size has been ensured.

${ }^{15}$ G. Lehmann and M. Taut, Phys. Status Solidi B 54, 469 (1972).

${ }^{16}$ J. Hama, M. Watanabe, and T. Kato, J. Phys.: Condens. Matter 2, 7445 (1990).

${ }^{17}$ J. Pedersen, C. Flindt, N. A. Mortensen, and A.-P. Jauho, 28th International Conference on the Physics of Semiconductors -
ICPS 2006, (AIP, Melville, NY, 2007), p. 821.

${ }^{18}$ J. Pedersen, Master's thesis, Technical University of Denmark, Kongens Lyngby, Denmark, 2007.

${ }^{19}$ N. A. Mortensen, Opt. Lett. 30, 1455 (2005).

${ }^{20}$ N. A. Mortensen, J. Eur. Opt. Soc., Rapid Publ. 1, 06009 (2006).

${ }^{21}$ L. I. Glazman, G. K. Lesovik, D. E. Khmelnitskii, and R. I. Shekter, JETP Lett. 48, 238 (1988).

${ }^{22}$ In practice, reliable single-electron filling may pose a serious experimental challenge which could require further optimization of the architecture presented here.

${ }^{23}$ G. M. Nikolopoulos, D. Petrosyan, and P. Lambropoulos, J. Phys.: Condens. Matter 16, 4991 (2004).

${ }^{24}$ G. M. Nikolopoulos, D. Petrosyan, and P. Lambropoulos, Europhys. Lett. 65, 297 (2004).

${ }^{25}$ M. Skorobogatiy, K. Saitoh, and M. Koshiba, Opt. Lett. 31, 314 (2006).

${ }^{26}$ M. Skorobogatiy, K. Saitoh, and M. Koshiba, Opt. Express 14, 1439 (2006).

${ }^{27}$ Another experimental challenge relates to the $R C$ switching time of the back gate which grows with length, making it harder to control on short time scales.

${ }^{28}$ D. P. DiVincenzo, D. Bacon, J. Kempe, G. Burkard, and K. B. Whaley, Nature (London) 408, 339 (2000).

${ }^{29}$ J. Pedersen, C. Flindt, N. A. Mortensen, and A.-P. Jauho, Phys. Rev. B 76, 125323 (2007).

${ }^{30}$ M. Helle, A. Harju, and R. M. Nieminen, Phys. Rev. B 72, 205329 (2005).

${ }^{31}$ Because we use an expansion in localized single-particle product states our method is most reliable for systems with several localized single-particle states. We consequently focus on relative antidot diameters above the single-level regime of the single defects $(d / \Lambda>0.42)$, see Fig. 3. 